\title{
BMJ Open Adapting the ToyBox obesity prevention intervention for use in Scottish preschools: protocol for a feasibility cluster randomised controlled trial
}

\author{
Stephen Malden, ${ }^{1}$ Adrienne R Hughes, ${ }^{1}$ Ann-Marie Gibson, ${ }^{1}$ Farid Bardid, ${ }^{2,3}$ \\ Odysseas Androutsos, ${ }^{4}$ Marieke De Craemer, ${ }^{3}$ Yannis Manios, ${ }^{4}$ \\ Carolyn Summerbell, ${ }^{5}$ Greet Cardon, ${ }^{3}$ John J Reilly ${ }^{1}$
}

To cite: Malden S, Hughes AR, Gibson A-M, et al. Adapting the ToyBox obesity prevention intervention for use in Scottish preschools: protocol for a feasibility cluster randomised controlled trial. BMJ Open 2018:8:e023707. doi:10.1136/ bmjopen-2018-023707

- Prepublication history for this paper is available online. To view these files, please visit the journal online (http://dx.doi. org/10.1136/bmjopen-2018023707).

Received 19 April 2018 Revised 12 September 2018 Accepted 20 September 2018

Check for updates

(C) Author(s) (or their employer(s)) 2018. Re-use permitted under CC BY-NC. No commercial re-use. See rights and permissions. Published by BMJ.

For numbered affiliations see end of article.

Correspondence to

Stephen Malden;

stephen.malden@strath.ac.uk

\section{ABSTRACT}

Introduction There is an increasing need for the adoption of effective preschool obesity prevention interventions to combat the high levels of early-childhood obesity in the UK. This study will examine the feasibility and acceptability of the adapted version of the ToyBox intervention-a preschool obesity prevention programme-for use in Scotland (ToyBox-Scotland). This will inform the design of a full-scale cluster randomised controlled trial (RCT).

Methods and analysis The ToyBox-Scotland intervention will be evaluated using a feasibility cluster RCT, which involves children aged 3-5years at six preschools in Glasgow, three randomly assigned to the intervention group and three to the usual-care control group. The original ToyBox intervention was adapted for the Scottish context using a coproduction approach. Within the 18-week intervention, physical activity and sedentary behaviour will be targeted in the preschool through environmental changes to the classroom, physical activity sessions and movement breaks. Parents will receive home activity packs every 3 weeks containing sticker incentives and interactive parent-child games that target sedentary behaviour, physical activity, eating/snacking and water consumption. As this is a feasibility study, parameters such as recruitment rates, attrition rates and SDs of outcome measures will be obtained which will inform a power calculation for a future RCT. Additional variables to be assessed include accelerometer-measured physical activity, sedentary behaviour and sleep, body mass index, home screen time, eating/snacking and water consumption. Outcomes will be assessed at baseline and 14-17 weeks later. Intervention fidelity will be assessed using questionnaires and interviews with parents and practitioners, observation and session delivery records.

Ethics and dissemination This study was granted ethical approval by the University of Strathclyde's School of Psychological Sciences and Health Ethics Committee. Results will be disseminated through publication in peerreviewed journals, presentation at conferences and in lay summaries provided to participants.

Trial registration number ISRCTN12831555.
Strengths and limitations of this study

- Site-specific adaptation of a previously successful intervention (ToyBox, which targets key obesogenic behaviours in early childhood) for use in another country (ToyBox Scotland).

- Culturally relevant, multicomponent intervention targeting the preschool and home setting.

- Coproduction approach used to involve stakeholders (ie, early years practitioners) in the intervention development and adaptation process.

- Use of both quantitative (eg, objective measures, observation and self-report) and qualitative (eg, logbook, interviews) methods to test feasibility.

- The study is limited by a short duration and a small number of clusters.

- Direct parental input is needed to further develop the home component of the intervention.

\section{INTRODUCTION}

Childhood obesity is a global public health problem, particularly in developed countries. ${ }^{12}$ Overweight and obesity rates for children under 10 years of age in Europe were reported to be approximately $20 \%$, with the UK having one of the highest levels of childhood obesity within this region. ${ }^{3-5}$ The rates reported for Scotland are particularly high, with at least $22.9 \%$ of $4-5$ years old being overweight or obese in 2016/2017. ${ }^{67}$

The causal factors contributing to childhood obesity are complex. ${ }^{8} 9$ However, a substantial evidence base has demonstrated that energy balance-related behaviours such as physical activity (PA), sedentary behaviour (SB) and the consumption of unhealthy drinks and snacks have a major influence on the development of childhood obesity. ${ }^{10-19}$ While efforts to address these 
energy balance-related behaviours are often focused around school-aged children, ${ }^{20}$ intervening at an earlier age is merited. Recent research has demonstrated that physical inactivity and SB in the early years tracks into later childhood and adolescence. ${ }^{21}$ Specifically, children who engage in healthy behaviours from an early age are more likely to maintain such behaviours throughout childhood, ${ }^{22}{ }^{23}$ potentially preventing the onset of obesity when it is perhaps more difficult to reverse. ${ }^{24}$

Results of obesity prevention interventions targeting preschool children (3-5 years old) are equivocal..$^{25}$ Some reported encouraging findings regarding weight-related outcomes, ${ }^{26-32}$ while others were ineffective. ${ }^{33-36}$ Interestingly, successful interventions tend to have similar characteristics in that they target multiple behaviours (eg, PA and diet) and/or multiple environments (eg, preschool and home). Two examples are the 14-week US Hip Hop to Health study ${ }^{28}$ and the year-long Spanish Ballabeina Study ${ }^{29}$ in which the preschool and home-based interventions led to significantly improved weight-related outcomes in comparison to control groups. A novel strength of the Hip Hop to Health study was that it improved weight-related outcomes in a hard-to-reach minority group. Such findings highlight the importance of focusing on obesity prevention efforts both at preschool and at home, and the benefit of adapting interventions in order to successfully engage specific population groups.

In Scotland, children between the ages of 3 and 5 years are entitled to 600 hours of free childcare per year, which the majority of children spend in a preschool setting. ${ }^{37}$ As these children still spend a substantial amount of time at home, it is important that interventions contain a homebased component in order to target obesity-related health behaviours across both environments.

ToyBox is one such intervention which was developed to prevent obesity in young children from varying backgrounds through the use of a multicomponent, evidence-based and theory-based, family-involved intervention implemented in preschools and homes across six European countries from 2012 to 2013. ${ }^{38}$ Extensive intervention development and site-specific adaptation was undertaken, detailed elsewhere. ${ }^{39-43}$ ToyBox aimed to improve energy balance-related behaviours through the delivery of teacher-led PA and SB sessions, promotion of water and healthy snacking/eating, environmental changes to the classroom, and parental education materials and parent-child activities. Evaluations of ToyBox have demonstrated that the intervention was feasible, while significant improvements in $\mathrm{PA}$ and SBs were observed. ${ }^{43}{ }^{44}$ In the Belgian cohort, children at intervention preschools significantly increased their vigorous PA $(\beta=1.47, p=0.03)$ and moderate-to-vigorous $\mathrm{PA}(\beta=1.27$, $\mathrm{p}=0.03$ ) from baseline to follow-up, while control participant's activity levels remained constant or decreased. ${ }^{45}$ Furthermore, while there were no significant interaction effects on SB for the total sample, among children who spent the most time in sedentary activities at baseline, a significant reduction in objectively measured sedentary time of $-4.17 \%$ was observed for the intervention group in comparison to the control group $(-0.41 \%) .{ }^{46}$ Process evaluation of the intervention has also identified important areas for consideration in any future expansion or adaptation of the intervention, such as the need for more active parental involvement. ${ }^{44}$ Due to the differences between preschools in Scotland/the UK and other European countries, the feasibility and efficacy of the programme cannot be assumed and must therefore be tested following appropriate adaptation. Interventions which are not appropriately adapted to the specific environment, often fail to have the desired impact. Therefore, extensive adaptation of intervention components and stakeholder involvement is becoming an increasingly common practice in intervention development and adaptation, ${ }^{47}$ in addition to feasibility testing prior to full-scale evaluation. ${ }^{48}$ The present feasibility cluster randomised controlled trial (RCT) aims to test the feasibility of the culturally adapted ToyBox intervention for use in Scotland (ToyBox-Scotland), a country with high childhood obesity rates. ${ }^{5}$ As this is a feasibility study, the primary aim will be to assess whether progression to a full-scale cluster RCT is merited through the evaluation of important aspects such as intervention fidelity and acceptability.

\section{METHODS}

This protocol has been prepared in accordance with the Standard Protocol Items: Recommendations for Interventional Trials statement. ${ }^{49} 50$

\section{Patient and public involvement}

This intervention was adapted with the assistance of an early years practitioner and staff at a local authority preschool. Results of the study will be disseminated to participants through a presentation to early years practitioners and a lay summary of results will be provided to parents.

\section{Study setting and participants}

Participants will be recruited from six local authority preschool settings in Glasgow. The city is one of the most socioeconomically deprived areas in Western Europe, with $34 \%-41 \%$ of the child population living in poverty. ${ }^{51}$ In 2015, at least $5.9 \%$ of 4-5years old in Glasgow were severely obese by body mass index (BMI $\geq 98$ th centile), ${ }^{51}$ with rates considerably higher among more deprived population groups. ${ }^{7}$

The intervention will be delivered to all children aged $3-5$ years in the participating preschools.

\section{Inclusion criteria}

- Preschool-level inclusion criteria: Glasgow City Council preschool settings (nurseries, early years centres or family learning centres) that are delivering the Scottish Curriculum for Excellence (for 3-18 years old) in accordance with Education Scotland's early learning and childcare frameworks and guidance. ${ }^{52}$ 
- Individual-level inclusion criteria: preschool year children aged 3-5years and their parents/caregivers who consent to both themselves and the child participating in data collection.

\section{Exclusion criteria}

- Children for whom parental/caregiver consent is not provided and children with health conditions which would affect their participation in the intervention.

\section{Recruitment and consent}

All local authority preschools in Glasgow $(n=112)$ were contacted via email by a City Council representative on behalf of the research team to provide information on the study, and request expressions of interest to participate. Eleven preschools expressed an interest. The majority of these preschools were located within the $20 \%$ most deprived areas in Scotland as defined by the Scottish Index of Multiple Deprivation (SIMD). The SIMD compiles data on a number of domains including education, crime, health, income and housing to develop an area-based indicator of deprivation for all neighbourhoods in Scotland. Six preschools with similar SIMD scores (all with low-medium deprivation scores), demographics and class size were invited to participate. A seventh preschool with similar characteristics to the study preschools agreed to assist with adaptation and coproduction of the intervention, which is discussed in more detail later. Preschool head teachers were provided with information sheets when approached to participate. All parents/caregivers of preschool year children aged 3-5 years at participating preschools received an information sheet detailing the intervention and data collection procedures via preschool staff, along with a consent form for their child's participation in data collection. All participating nurseries will receive $£ 200$ to offset the additional costs of taking part. Children will receive a colouring book for participation in data collection.

\section{Intervention}

The original ToyBox-study used various theories of health behaviour change including social cognitive theory, theory of planned behaviour and health belief model to influence energy balance-related behaviours both in preschool settings and with families in the home. ${ }^{39}$ The programme was developed using the PRECEDE-PROCEED mode ${ }^{53}$ and the intervention mapping protocol. ${ }^{54}$ The overall aim of the intervention was to improve children's health behaviours in relation to obesity and it has subsequently been implemented in $>300$ preschools across Europe (Greece, Spain, Germany, Poland, Bulgaria and Belgium). The ToyBox-study had four main areas of focus (ie, PA, SB, healthy snacking and water consumption) as detailed in table 1. Classroom activities focused on each targeted behaviour for four consecutive weeks, before the cycle was repeated for a further 2 weeks for each behaviour. Classroom guides were designed for each targeted health behaviour containing instructions for the set-up and delivery of activities and workshops, in addition to interactive stories involving cartoon animal role models. Practitioners were given autonomy in choosing activities from the provided classroom guides to deliver throughout the day and week. However, they were encouraged to deliver sessions totalling 1 hour per week, and to gradually introduce more advanced activities from the classroom guides as the intervention progressed. Teachers were provided with training on the programme delivery before starting the intervention.

The family-based component of the intervention involved the provision of tip cards, posters and newsletters to parents/caregivers. Distribution of these materials coincided with the particular behaviour being focused on at the preschool. Tip cards offered suggestions of ways to improve each health behaviour in the home, posters featured cartoon characters from the classroom guides performing behaviours, while newsletters reinforced health messages.

\section{Adaptation of ToyBox for use in Scotland}

ToyBox-Scotland follows the same principles and procedures as the original ToyBox-study. However, adaptations have been made to reflect the cultural, legislative and infrastructural differences between preschools in Scotland and the other European countries included in the original ToyBox-study, a practice supported by intervention development literature. ${ }^{39}$ During initial planning of ToyBox-Scotland, regular meetings were held with Glasgow City Council's education services team where the content and relevance of the original intervention materials were discussed, and the components of the intervention were assessed to ensure they aligned with key healthy weight policies for Scotland. ${ }^{55} 56$ A major outcome of these meetings was that the council strongly felt that the diet component of the intervention would be rendered obsolete in the Scottish preschool setting due to nearly all local authority preschools prohibiting children consuming 'junk food' or sugar-sweetened beverages on the premises. This was discussed in a workshop with early years practitioners, who confirmed that only healthy snacks, water and milk were permitted in their preschools. However, the practitioners believed that

Table 1 Original ToyBox-study intervention structure for classroom and home behaviour focus ${ }^{74}$

First focus

Repetition

\begin{tabular}{|c|c|c|c|c|c|c|c|}
\hline 4 weeks & 4 weeks & 4 weeks & 4 weeks & 2 weeks & 2 weeks & 2 weeks & 2 weeks \\
\hline $\begin{array}{l}\text { Water } \\
\text { drinking }\end{array}$ & $\begin{array}{l}\text { Physical } \\
\text { activity }\end{array}$ & $\begin{array}{l}\text { Eating and } \\
\text { snacking }\end{array}$ & $\begin{array}{l}\text { Sedentary } \\
\text { behaviour }\end{array}$ & $\begin{array}{l}\text { Water } \\
\text { drinking }\end{array}$ & $\begin{array}{l}\text { Physical } \\
\text { activity }\end{array}$ & $\begin{array}{l}\text { Eating and } \\
\text { snacking }\end{array}$ & $\begin{array}{l}\text { Sedentary } \\
\text { behaviour }\end{array}$ \\
\hline
\end{tabular}


eating, snacking and water consumption needed to be addressed in the home environment. Practitioners were less certain about the levels of PA and SB in preschools. Some believed that children were 'always on the move', and that PA was not an issue in Scottish preschools, while others thought more could be done to keep children active. Again, there was a consensus that both behaviours were major issues at home. In order to gain more insight into this, a small needs assessment study was conducted (results unpublished) which involved a sample of 15 preschoolers wearing an ActivPAL accelerometer for three consecutive days while at preschool and at home to measure PA and SB. The findings suggest that considerable periods of inactivity exist both at preschool and at home, which is in line with recent research on SB and PA levels in preschoolers. ${ }^{1021}$ This highlights the need to address these behaviours in early childhood as noted by the 2016 WHO commission report on ending childhood obesity ${ }^{57}$ and the newly published 24 hours movement guidelines. ${ }^{58}$ Nursery visits were also conducted in four preschools across Glasgow to observe children's PA and SB. While outdoors, children were generally active, however in the classroom, activity levels varied. A number of opportunities to reduce SB by making changes to the classroom environment were identified. ${ }^{39}$

Involving stakeholders in the development and adaptation of interventions is a crucial step in ensuring a programme is acceptable and practical for those who will be responsible for delivering it. ${ }^{59}$ It was therefore deemed necessary to involve preschool staff in the development and adaptation of ToyBox-Scotland, and a seventh preschool was recruited to assist with intervention adaptation following the principles of coproduction, ${ }^{60}$ before the cluster RCT was undertaken in the other six preschools. An experienced early years practitioner from this preschool assisted by reviewing all of the classroom guides from the original ToyBox intervention, trialling out each activity with the children, and matching the ToyBox-Scotland programme with the Scottish preschool education curriculum. A number of meetings were held with the early years practitioner during this period, and together with the research team, a number of changes to the classroom guides and materials were agreed on. One of the main changes was to ensure the included activities were child led, rather than practitioner led, which is in keeping with the curriculum of preschool education in Scotland. Child-led learning in the context of the Scottish curriculum ensures that children are given the freedom to interact with learning tasks and materials with minimal adult direction. ${ }^{37}$ Therefore, activities which did not allow for this were either removed or adapted. Out of $26 \mathrm{PA}$ sessions from the original ToyBox-study, 11 were selected as they offered the most opportunities for child-led learning while still engaging with the PA elements. These were also the activities that the children found easiest to engage with, reflecting the younger age of Scottish preschool children compared with children from other European countries. ${ }^{61}$ The selected activities were also included as they were the most likely to encourage MVPA as opposed to lower intensity PA throughout the session. The SB activities (consisting of 'movement breaks', 'movement corners' and classroom activities) were also trialled out. A number of the more cognitively/physically complex movement breaks and classroom activities were removed following recommendations from early years practitioners as the activities tended to break down or children would lose focus during delivery. The objective of the movement breaks is to interrupt prolonged periods of sitting, but these are shorter than the PA sessions. Finally, extensive revisions to the classroom guides and materials were undertaken to ensure the language used is relevant to the Scottish context. The structure of the adapted ToyBox-Scotland intervention is summarised in table 2.

Although the original ToyBox intervention included a home component, subsequent evaluations of the programme concluded that more active parental involvement was needed. ${ }^{446}$ ToyBox-Scotland will adopt the original home materials, but will also provide parent-child homework tasks, designed to increase $\mathrm{PA}$, reduce $\mathrm{SB}$ and encourage healthy snacking and water consumption over junk food and sugar-sweetened beverage consumption. A Scottish-themed sticker chart was developed along with animal stickers, which parents award to the children for completion of the homework activities. The use of sticker incentives is cost-effective and has been used in successful interventions to change health behaviours with this age group. ${ }^{62-64}$ While the original ToyBox study used passive parental education techniques such as tip cards and newsletters, ToyBox-Scotland will use interactive games and activities which require active involvement from parents and children, a recommendation of the original study. The ToyBox-Scotland intervention will run for a total of 18 weeks from March to June 2018. Control preschools will continue to follow the standard curriculum during this period. Figure 1 outlines the differences between the original and adapted intervention and intervention development processes.

Table 2 Adapted ToyBox-Scotland intervention structure

\begin{tabular}{|c|c|c|c|c|c|c|c|c|}
\hline & \multicolumn{4}{|l|}{ First focus } & \multicolumn{4}{|l|}{ Repetition } \\
\hline & 3 weeks & 3 weeks & 3 weeks & 3 weeks & 2 weeks & 2 weeks & 1 week & 1 week \\
\hline Preschool & PA & SB & PA & SB & PA & SB & PA & SB \\
\hline
\end{tabular}

ES, eating and snacking; PA, physical activity; SB, sedentary behaviour; WC, water consumption. 


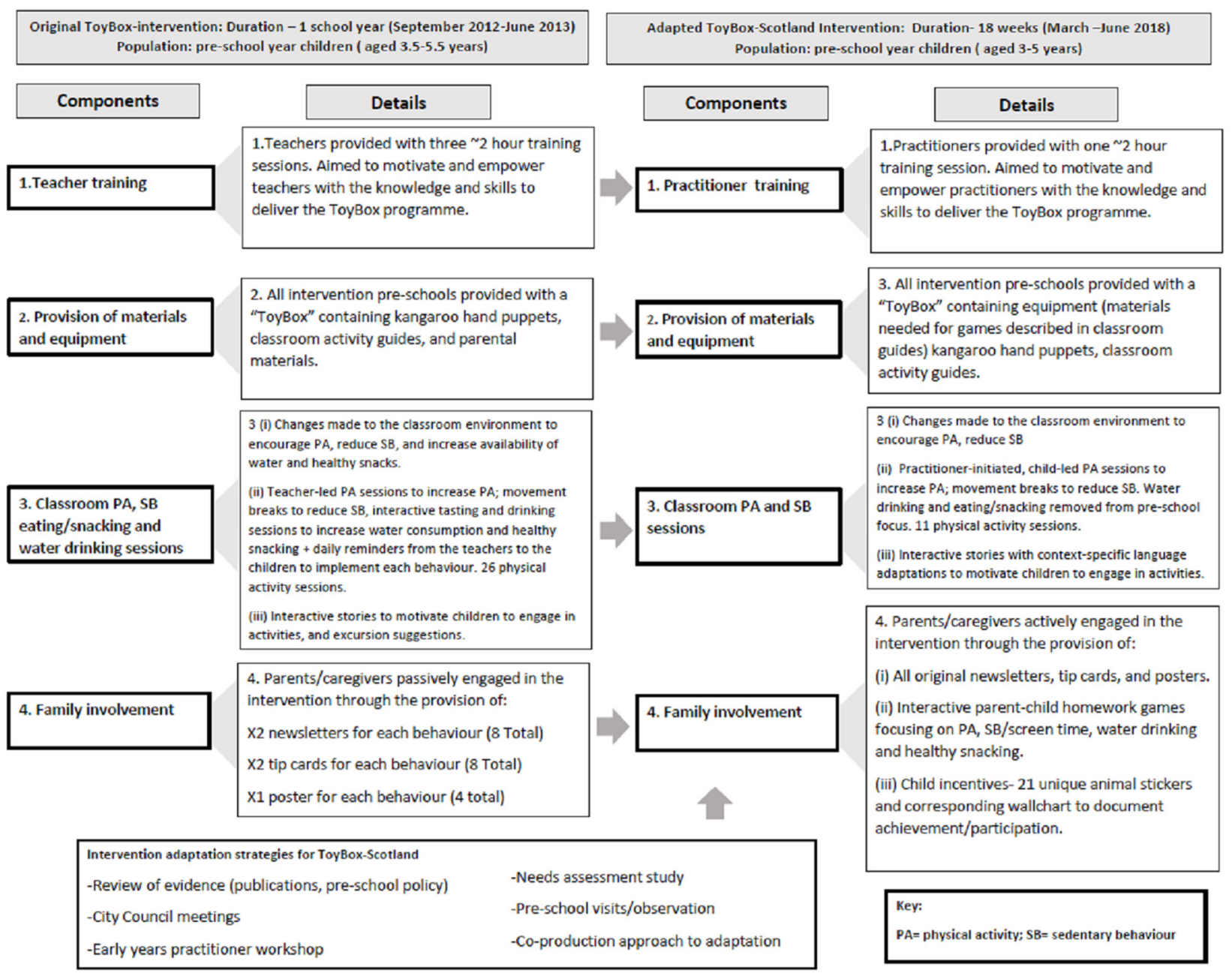

Figure 1 The differences between the original ToyBox programme and the adapted ToyBox Scotland programme.

\section{Practitioner training}

Early years practitioners in the intervention group will receive a 2-hour 30 min training session prior to intervention commencement, delivered by the lead researcher and the early years practitioner involved in coproducing ToyBox-Scotland. The session will cover guidelines for designing a movement-friendly classroom environment, delivery of the $\mathrm{PA} / \mathrm{SB}$ components, use of the practitioner logbook and a briefing on the content and timing of the parental component of the intervention. During the first 3 weeks of intervention delivery, the lead researcher and coproducing early years practitioner will visit the preschools to assist with any issues, and observe delivery of the programme to ensure it is being delivered as intended.

Parents will be provided with an introductory pack at the start of the intervention detailing what ToyBox-Scotland is and how it relates to them. They will be given instructions on how to use the wallchart and stickers to incentivise their child's behaviour, and short instructions for each homework game will be provided to parents via the early years practitioners.

\section{Outcomes}

Data collection will take place at baseline and 14-17 weeks later. The main outcome of the study is to determine the feasibility and acceptability of the ToyBox-Scotland intervention within Scottish preschools, which will involve the assessment of the following:

1. Recruitment rates (preschool and individual level).

2. Intervention fidelity (measured by assessing the practitioner's logbook of the number of sessions conducted per week, and structure followed in relation to classroom guides. Observation of delivery will also be undertaken, as will qualitative interviews with practitioners and parents on completion of the intervention).

3. Preschool and participant attrition rates.

4. Acceptability of the methods used for measuring the intervention effect (assessment of whether children wear the ActivPAL for the desired time period, and through questionnaire response rates).

Additional variables will be measured to assess the direction of intervention effect, and to calculate SD and intracluster correlation coefficients to inform sample size calculations for a full-scale cluster RCT. Other 
outcomes, measured at baseline and follow-up, are as follows:

1. Preschool and home PA: Children will be fitted with the ActivPAL accelerometer (PAL technologies, Glasgow UK) for seven consecutive days, including oneweekend. Mean minutes spent in PA will be recorded, both moderate-to-vigorous PA and total PA.

2. Preschool and home sedentary time: Total time spent sitting/lying and interruption of time spent sitting/ lying will be assessed using the ActivPAL. Previous studies have demonstrated the ActivPAL's suitability for measuring sedentary time and PA with this age group $^{6566}$ in addition to its high performance at measuring stepping when compared with other accelerometers. $^{67}$

3. Total night-time sleep: ActivPALs will be used to assess night-time sleep, with sleep time defined as the time point (to the second) where no movement from the sitting/lying axis plane of movement is detected by the device for a minimum of $120 \mathrm{~min}$. The devices have been successfully used to accurately estimate total sleep time and interruption of sleep in previous research. ${ }^{68}$

4. BMI: Children's height and weight will be measured by a trained researcher (SM) using a stadiometer (Marsden, UK) and electronic scales (Tanita, Amsterdam, Netherlands), respectively. Measurements will be taken to the nearest $0.1 \mathrm{~cm}$ for height and $0.1 \mathrm{~kg}$ for weight, with shoes removed. Height and weight measures will be taken twice and the average will be calculated. zBMI will be calculated from the height and weight measures, with obesity and overweight defined as 95th and 85 th percentiles, respectively, in accordance with the UK 1990 growth reference data. ${ }^{69}$

5. Body composition: Fat mass and fat-free mass will be estimated via supine arm-to-leg bioelectrical impedance analysis using the Bodystat 1500 . Measures will be taken by a trained researcher (SM) in the presence of a practitioner. Detailed descriptions of the methodological procedures and validation of this measure in children are available elsewhere. ${ }^{70}$ Measures will be taken twice and averaged.

6. Home eating/snacking, water consumption and screen time: Questionnaires will be completed by parents/caregivers of participating children and will include questions relating to eating/snacking behaviour (such as fruit/vegetable consumption, refined sugar consumption), daily water intake, daily PA and time spent using screen devices. During baseline data collection, these questionnaires will also contain relevant questions relating to demographics. The questionnaires were developed for use in the original ToyBoxstudy and have been adapted for ToyBox-Scotland. Specifically, both questionnaires were shortened and the language altered following recommendations from early years practitioners (eg, 'kindergarten' was changed to 'nursery'). No previously unvalidated items were added. Details regarding the original questionnaire development, test-retest reliability and validity are published elsewhere. ${ }^{71}$

\section{Process evaluation}

A process evaluation will be conducted to assess intervention fidelity and acceptability as follows:

Intervention fidelity-preschool component: A researcher will observe the delivery of two sessions of PA and SB at each preschool. The researcher will assess if the delivered sessions match the instructions provided in the classroom guides. Specifically, details on the duration of the sessions, proportion of children who participate, intensity of PA sessions and practitioner instruction/ engagement will be recorded. Practitioners will complete logbooks which will detail which specific sessions/activities were delivered each week and note any issues.

Intervention acceptability-preschool component: Semistructured interviews will be conducted at the preschools with a sample of early years practitioners who deliver the intervention. Interviews will primarily focus on the barriers and facilitators to delivery and identify any areas for improvement in the event that a full-scale trial is implemented. Practitioners will also be asked to complete a pre and postintervention questionnaire, which along with interview and logbook data will be used for data triangulation.

Intervention fidelity-home component: Practitioners will document the proportion of eligible children who are given home material packs and who take them home from the preschool. End of intervention feedback questionnaires will be provided to parents in the final homework packs. The questionnaires will seek to determine the level of engagement with each block of materials, parental perceptions of the homework tasks/materials and perceived changes to behaviour or enhanced knowledge as a result of the homework tasks/intervention materials.

Intervention acceptability-home component: In addition to the end of study questionnaire, a sample of parents will also participate in a semi-structured interview to gain an in-depth understanding of their views and experiences of engaging with the intervention materials. Interview topics will mainly focus on parent/child perceptions, barriers/facilitators to participation and ways in which the content or delivery of the materials can be improved on. Interviews will be conducted either at the preschool or by telephone. This information will be used to inform further adaptations of the home component of the intervention. A timeline of participant's involvement in the study is detailed in figure 2 .

\section{Ethics and dissemination}

Any amendments to the study protocol will be submitted for ethical approval prior to implementation. Informed consent will be obtained from all participants via parental consent forms. Verbal assent will be sought from children prior to their enrolment in the study. Findings of the study will be disseminated via publications in 


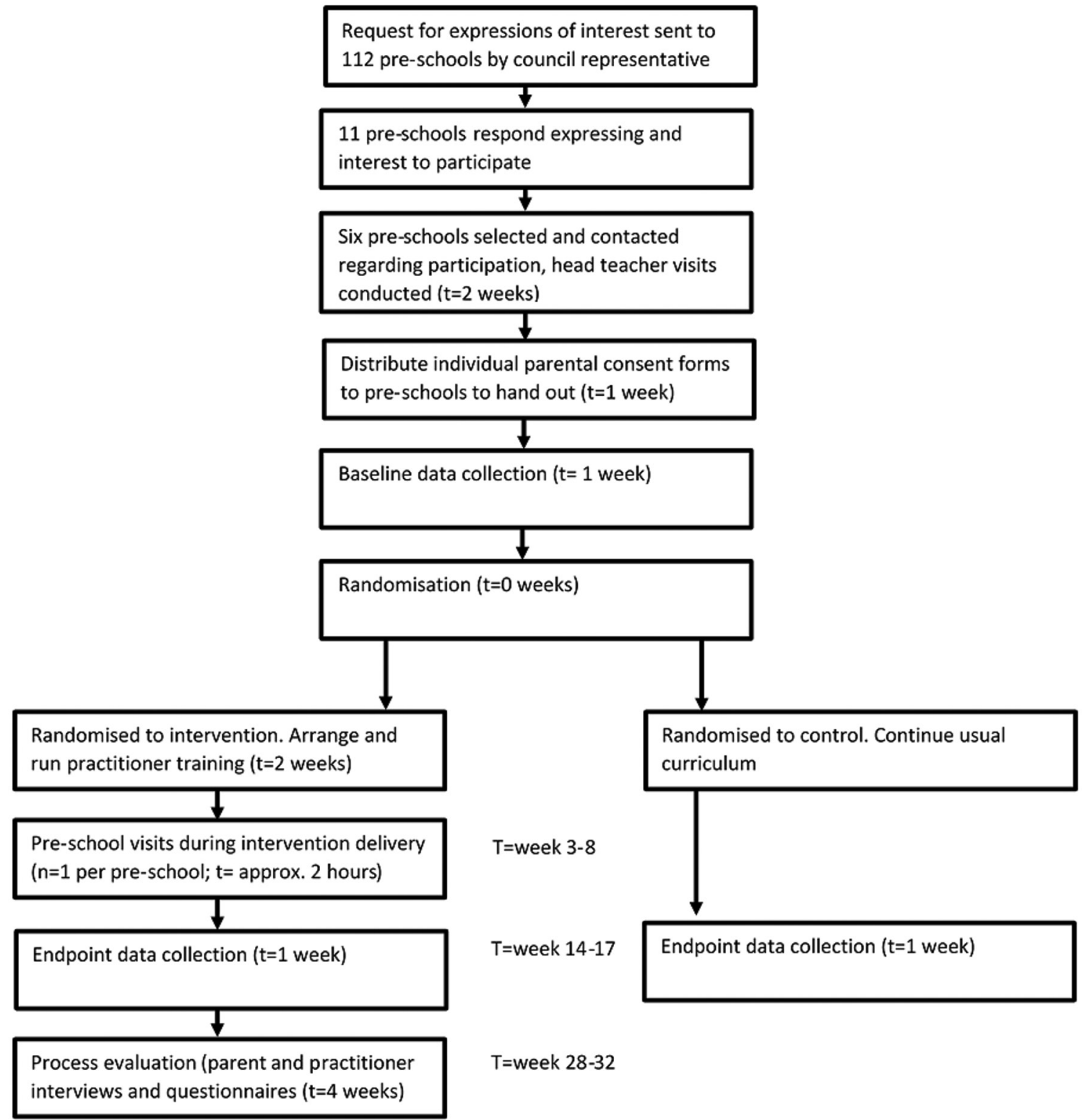

Figure 2 Participant timeline detailing timings and length of participant contact periods.

peer-reviewed journals, conference presentations and lay summary reports/presentations which will be given to parents/caregivers and preschool staff who participate in the study.

\section{Sample size}

As this is a feasibility study, one of the main objectives is to collect appropriate data to inform a power calculation for a future full-scale cluster RCT. Therefore, no sample size calculation was undertaken for this study, and the inclusion of six preschools is unlikely to be sufficient for any definitive conclusions to be drawn regarding the intervention's effect on health and behaviour outcomes. Rather, the sample will be sufficient to measure important feasibility parameters in a sample of preschools that are representative of the Glasgow City preschool environment. Furthermore, feasibility testing is recommended as one of the key elements of the intervention development and evaluation process outlined by the UK Medical Research Council. ${ }^{48}$

\section{Randomisation}

Randomisation will occur following completion of baseline data collection, and will be conducted by a blinded independent researcher. Simple randomisation will be undertaken at the preschool level, with three preschools randomised to intervention (ToyBox-Scotland) arm and three to the control (usual curriculum) arm. As all recruited preschools are of a similar socioeconomic status and size, no pairing will be undertaken prior to randomisation.

\section{Data management}

Data will be stored on the University of Strathclyde's centralised secure data storage system. Only the immediate research team will have access to raw data. Parental 
questionnaires will be kept by head teachers after completion and returned to the researcher. Consent forms will be stored separately from participant data, and a unique identifier code will be assigned to each participant. Data will be stored for a maximum of 5 years before being securely destroyed. Data from interviews will be deleted immediately from voice recorders after the transcription, with pseudonyms used in all reports in place of participant's names. Data will be available in anonymised format from the University of Strathclyde institutional repository. All data collection and storage procedures will be general data protection regulation compliant.

\section{Analysis}

A descriptive analysis will summarise the findings in relation to the feasibility and acceptability parameters of interest such as the proportion of children measured at baseline and follow-up to calculate recruitment and attrition rates. Data collected will be used to calculate the SD of the outcome measures and the sample sizes needed to detect a significant difference in a full-scale trial. Summary statistics will be applied to the outcome measures, with results presented as means $( \pm \mathrm{SD})$, and where appropriate ORs $(95 \% \mathrm{CI})$. Level of significance will be set at 0.05. Subgroup analysis will be performed to determine differences in recruitment and retention rates by socioeconomic status and direction of intervention effects by socioeconomic status, sex and weight status. However, due to the small sample size anticipated, these analyses will be exploratory in nature, and will be used to inform a future trial rather than to draw definitive conclusions regarding the effectiveness of the intervention. Participants who drop out will also be compared with those who complete the study, to determine if any significant differences exist. Data analysis will be conducted using IBM SPSS v25 statistical analysis software.

Interview data will be transcribed verbatim prior to thematic analysis. Data will be coded by two researchers independently, and themes will be formed from the identified codes that summarise the participant's responses. Data will be entered into NVivo V.10 qualitative data analysis software to assist with coding and development of themes.

\section{DISCUSSION}

This paper provides a description of the protocol for the ToyBox-Scotland preschool obesity prevention intervention trial. The original ToyBox-study successfully improved key energy balance-related behaviours among European preschool children, ${ }^{44}$ and has now been adapted for use in the Scottish preschool setting. By using a coproduction approach to adapt the programme, it is hoped that the intervention will be more culturally relevant and userfriendly for the practitioners who will be responsible for its delivery. Considerable adaptation has also been applied to the home component of the intervention in an effort to increase parental engagement. Adapting successful interventions for use in other settings is becoming an increasingly common practice in child health research. ${ }^{72}$ However, for success to translate from one setting to the other, adequate context-specific adaptations must be made to ensure the intervention can function as intended and still meets its desired aims. ${ }^{73}$ The study outlined in this protocol aims to test whether the ToyBox-Scotland obesity prevention intervention is feasible and acceptable in the Scottish preschool and home setting, and to determine whether progression to a full-scale cluster RCT is recommended.

\section{Trial status}

Study status as of 10/04/2018: Ethical approval has been granted for the study. All project funding is secured, and all preschool staff have been recruited. Child/parent recruitment was completed in February 2018. Baseline data collection was completed in late February 2018. Practitioner training took place early March 2018, and the intervention began in early March 2018. Follow-up data collection will take place in June 2018.

\section{Trial governance}

The principal investigators (ARH and JJR) are responsible for overseeing the study. The study manager (SM) is responsible for liaising with study participants, coordinating data collection and data management/storage. ARH, A-MG and FB will advise on specific aspects of the study including recruitment, practitioner training, data analysis and process evaluation procedures. Any changes to the study protocol will be discussed before the trial registry is updated.

\section{Safety procedures}

All preschool staff are first aid trained, and preschools have their own health and safety policies, which ToyBox-Scotland does not breach. In the event of an accident occurring as a direct consequence of participation in the study, (no high-risk activities were identified by risk assessment during ethics application), preschool staff will report this to the research team and appropriate measures will be taken according to existing policies.

\section{Author affiliations}

${ }^{1}$ Physical Activity for Health Group, School of Psychological Sciences and Health, University of Strathclyde, Glasgow, UK

${ }^{2}$ School of Education, University of Strathclyde, Glasgow, UK

${ }^{3}$ Department of Movement and Sports Sciences, Ghent University, Ghent, UK ${ }^{4}$ Department of Nutrition and Dietetics, School of Health Science and Education, Harokopio University, Athens, Greece

${ }^{5}$ Department of Sport and Exercise Sciences, Durham University, Durham City, UK

Acknowledgements The authors thank Jacqui Glover and all staff, parents and children at Sighthill nursery for their assistance with intervention adaptation, and Miruna-Diana Mateescu for assisting with the design of the wallchart and stickers.

Contributors SM was responsible for participant recruitment and data collection. SM prepared the first draft of the manuscript. JJR, ARH, A-MG, FB, MD, OA, YM, CS and GC made substantial contributions to the second draft of the manuscript. All authors were involved in the design of the study and development of intervention materials.

Funding This work was supported by The Cunningham Trust. 
Competing interests None declared.

Patient consent Not required.

Ethics approval This study was granted ethical approval by the University of Strathclyde's School of Psychological Sciences and Health Ethics Committee.

Provenance and peer review Not commissioned; externally peer reviewed.

Open access This is an open access article distributed in accordance with the Creative Commons Attribution Non Commercial (CC BY-NC 4.0) license, which permits others to distribute, remix, adapt, build upon this work non-commercially, and license their derivative works on different terms, provided the original work is properly cited, appropriate credit is given, any changes made indicated, and the use is non-commercial. See: http://creativecommons.org/licenses/by-nc/4.0/.

\section{REFERENCES}

1. Abarca-Gómez L, Abdeen ZA, Hamid ZA, et al. Worldwide trends in body-mass index, underweight, overweight, and obesity from 1975 to 2016: a pooled analysis of 2416 population-based measurement studies in 128.9 million children, adolescents, and adults. Lancet 2017;390:2627-42

2. Ogden CL, Carroll MD, Kit BK, et al. Prevalence of childhood and adult obesity in the United States, 2011-2012. JAMA 2014;311:806-14.

3. Ahrens W, Pigeot I, Pohlabeln $\mathrm{H}$, et al. Prevalence of overweight and obesity in European children below the age of 10. Int $J$ Obes 2014;38:S99-107.

4. Wijnhoven TM, van Raaij JM, Spinelli A, et al. WHO European Childhood Obesity Surveillance Initiative 2008: weight, height and body mass index in 6-9-year-old children. Pediatr Obes 2013;8:79-97.

5. Ng M, Fleming T, Robinson M, et al. Global, regional, and national prevalence of overweight and obesity in children and adults during 1980-2013: a systematic analysis for the Global Burden of Disease Study 2013. Lancet 2014;384:766-81.

6. Scottish Government. Health of Scotland's Population- Obesity. Secondary health of Scotland's population- obesity. 2017. https:// www.gov.scot/Topics/Statistics/Browse/Health/TrendObesity

7. National Health Service Scotland. Body mass index of primary 1 children in Scotland school year 2016/17: National Health Service Scotland, 2017.

8. McGlashan J, Johnstone M, Creighton D, et al. Quantifying a Systems Map: Network Analysis of a Childhood Obesity Causal Loop Diagram. PLoS One 2016;11:e0165459.

9. Orr MG, Kaplan GA, Galea S. Neighbourhood food, physical activity, and educational environments and black/white disparities in obesity: a complex systems simulation analysis. J Epidemiol Community Health 2016;70:jech-2015-205621.

10. Saldanha-Gomes C, Heude B, Charles MA, et al. Prospective associations between energy balance-related behaviors at 2 years of age and subsequent adiposity: the EDEN mother-child cohort. Int $J$ Obes 2017;41:38-45.

11. Janssen I, Leblanc AG. Systematic review of the health benefits of physical activity and fitness in school-aged children and youth. Int $J$ Behav Nutr Phys Act 2010;7:40.

12. Jiménez-Pavón D, Kelly J, Reilly JJ. Associations between objectively measured habitual physical activity and adiposity in children and adolescents: Systematic review. Int $J$ Pediatr Obes 2010;5:3-18.

13. Te Morenga L, Mallard S, Mann J. Dietary sugars and body weight: systematic review and meta-analyses of randomised controlled trials and cohort studies. BMJ 2012;346:e7492.

14. Basterfield L, Pearce MS, Adamson AJ, et al. Physical activity, sedentary behavior, and adiposity in English children. Am J Prev Med 2012;42:445-51.

15. Cardon G, De Bourdeaudhuij I, lotova V, et al. Health related behaviours in normal weight and overweight preschoolers of a large Pan-European sample: the ToyBox-study. PLoS One 2016;11:e0150580.

16. Ekelund U, Luan J, Sherar LB, et al. Moderate to vigorous physical activity and sedentary time and cardiometabolic risk factors in children and adolescents. JAMA 2012;307:704-12.

17. Frantsve-Hawley J, Bader JD, Welsh JA, et al. A systematic review of the association between consumption of sugar-containing beverages and excess weight gain among children under age 12. J Public Health Dent 2017;77 Suppl 1:S43-S66.

18. Hooper L, Abdelhamid A, Moore HJ, et al. Effect of reducing total fat intake on body weight: systematic review and meta- analysis of randomised controlled trials and cohort studies. BMJ 2012;345:e7666.

19. Tremblay MS, LeBlanc AG, Kho ME, et al. Systematic review of sedentary behaviour and health indicators in school-aged children and youth. Int J Behav Nutr Phys Act 2011;8:98

20. Wang Y, Wu Y, Wilson RF, et al. Childhood obesity prevention programs: comparative effectiveness review and meta-analysis. Rockville (MD): Agency for Healthcare Research and Quality (US), 2013.

21. Jones RA, Hinkley T, Okely AD, et al. Tracking physical activity and sedentary behavior in childhood: a systematic review. Am J Prev Med 2013:44:651-8.

22. Skinner JD, Carruth BR, Wendy B, et al. Children's food preferences: a longitudinal analysis. J Am Diet Assoc 2002;102:1638-47.

23. Moore LL, Gao D, Bradlee ML, et al. Does early physical activity predict body fat change throughout childhood? Prev Med 2003;37:10-17.

24. Nader PR, Huang TT, Gahagan S, et al. Next steps in obesity prevention: altering early life systems to support healthy parents, infants, and toddlers. Child Obes 2012;8:195-204.

25. Waters E, de Silva-Sanigorski A, Hall BJ, et al. Interventions for preventing obesity in children. Cochrane Database Syst Rev 2011:Cd001871.

26. Annesi JJ, Smith AE, Tennant GA. Reducing high BMI in African American preschoolers: effects of a behavior-based physical activity intervention on caloric expenditure. South Med J 2013;106:456-9.

27. de Silva-Sanigorski AM, Bell AC, Kremer P, et al. Reducing obesity in early childhood: results from Romp \& Chomp, an Australian community-wide intervention program. Am J Clin Nutr 2010;91:831-40.

28. Fitzgibbon ML, Stolley MR, Schiffer L, et al. Two-year follow-up results for Hip-Hop to Health Jr.: a randomized controlled trial for overweight prevention in preschool minority children. $J$ Pediatr 2005;146:618-25.

29. Niederer I, Bürgi F, Ebenegger V, et al. Effects of a lifestyle intervention on adiposity and fitness in overweight or low fit preschoolers (Ballabeina). Obesity 2013;21:E287-93.

30. Yin Z, Parra-Medina D, Cordova A, et al. Míranos! Look at us, we are healthy! An environmental approach to early childhood obesity prevention. Child Obes 2012;8:429-39.

31. Eliakim A, Nemet D, Balakirski Y, et al. The effects of nutritionalphysical activity school-based intervention on fatness and fitness in preschool children. J Pediatr Endocrinol Metab 2007;20:711-8.

32. Alkon A, Crowley AA, Neelon SE, et al. Nutrition and physical activity randomized control trial in child care centers improves knowledge, policies, and children's body mass index. BMC Public Health 2014:14:215.

33. Birken CS, Maguire J, Mekky M, et al. Office-based randomized controlled trial to reduce screen time in preschool children. Pediatrics 2012;130:1110-5.

34. Dennison BA, Russo TJ, Burdick PA, et al. An intervention to reduce television viewing by preschool children. Arch Pediatr Adolesc Med 2004;158:170-6.

35. Mo-suwan L, Pongprapai S, Junjana C, et al. Effects of a controlled trial of a school-based exercise program on the obesity indexes of preschool children. Am J Clin Nutr 1998;68:1006-11.

36. Winter SM, Sass DA. Healthy \& ready to learn: examining the efficacy of an early approach to obesity prevention and school readiness. Journal of Research in Childhood Education 2011;25:304-25.

37. Scottish Government. Early learning and childcare. Secondary Early learning and childcare. 2017. http://www.gov.scot/Topics/People/ Young-People/early-years/parenting-early-learning/childcare

38. Manios Y. The 'ToyBox-study' obesity prevention programme in early childhood: an introduction. Obes Rev 2012;13:1-2.

39. Manios Y. ToyBox-study group. Methodological procedures followed in a kindergarten-based, family-involved intervention implemented in six European countries to prevent obesity in early childhood: the ToyBox-study. Obes Rev 2014;15(Suppl 3):1-4.

40. Payr A, Birnbaum J, Wildgruber A, et al. Concepts and strategies on how to train and motivate teachers to implement a kindergartenbased, family-involved intervention to prevent obesity in early childhood. The ToyBox-study. Obes Rev 2014;15(Suppl 3):40-7.

41. Duvinage K, Ibrügger S, Kreichauf S, et al. Developing the intervention material to increase physical activity levels of European preschool children: the ToyBox-study. Obes Rev 2014;15(Suppl 1):27-39.

42. Nixon CA, Moore HJ, Douthwaite W, et al. Identifying effective behavioural models and behaviour change strategies underpinning preschool- and school-based obesity prevention interventions aimed at 4-6-year-olds: a systematic review. Obes Rev 2012;13(Suppl 1):106-17. 
43. Androutsos O, Katsarou C, Payr A, et al. Designing and implementing teachers' training sessions in a kindergarten-based, family-involved intervention to prevent obesity in early childhood. The ToyBox-study. Obes Rev 2014;15(Suppl 3):48-52.

44. Latomme J, Cardon G, De Bourdeaudhuij I, et al. Effect and process evaluation of a kindergarten-based, family-involved intervention with a randomized cluster design on sedentary behaviour in 4- to 6- year old European preschool children: The ToyBox-study. PLoS One 2017; 12:e0172730.

45. De Craemer M, De Decker E, Verloigne M, et al. The effect of a kindergarten-based, family-involved intervention on objectively measured physical activity in Belgian preschool boys and girls of high and low SES: the ToyBox-study. Int J Behav Nutr Phys Act 2014;11:38.

46. De Craemer M, De Decker E, Verloigne M, et al. The effect of a cluster randomised control trial on objectively measured sedentary time and parental reports of time spent in sedentary activities in Belgian preschoolers: the ToyBox-study. Int J Behav Nutr Phys Act 2016;13:1.

47. Sutherland RL, Nathan NK, Lubans DR, et al. An RCT to facilitate implementation of school practices known to increase physical activity. Am J Prev Med 2017;53:818-28.

48. Craig P, Dieppe P, Macintyre S, et al. Developing and evaluating complex interventions: the new Medical Research Council guidance. BMJ 2008;337:a1655.

49. Chan AW, Tetzlaff JM, Altman DG, et al. SPIRIT 2013 statement: defining standard protocol items for clinical trials. Ann Intern Med 2013;158:200-7.

50. Chan AW, Tetzlaff JM, Gøtzsche PC, et al. SPIRIT 2013 explanation and elaboration: guidance for protocols of clinical trials. BMJ 2013;346:e7586.

51. Scottish Cities. Secondary Scottish Cities. Understanding Glasgow: The Glasgow Indicators Project. 2015. http://www. understandingglasgow.com/indicators/children/poverty/scottish_ cities

52. Education Scotland. Early learning and childcare (ELC). Secondary Early learning and childcare (ELC). 2016. https://education.gov. scot/scottish-education-system/Early\%20learning\%20and\% 20childcare\%20(ELC)

53. Green LW, Kreuter MW. Health program planning: an educational and ecological approach. New York: McGraw-Hill, 2005.

54. Kok G, Schaalma H, Ruiter RA, et al. Intervention mapping: protocol for applying health psychology theory to prevention programmes. $J$ Health Psychol 2004;9:85-98.

55. Scottish Government. A healthier future -Scotland's Diet \& Healthy weight delivery plan. Edinburgh: Scottish Government, 2018.

56. Scottish Government. A more active Scotland: Scotland's physical activity delivery plan. Edinburgh: Scottish Government, 2018.

57. WHO. Report of the commission on ending childhood obesity. Geneva: WHO, 2016.

58. Tremblay MS, Chaput JP, Adamo KB, et al. Canadian 24-Hour Movement Guidelines for the Early Years ( $0-4$ years): an integration of physical activity, sedentary behaviour, and sleep. BMC Public Health 2017; $17: 874$
59. Wight $\mathrm{D}$, Wimbush $\mathrm{E}$, Jepson $\mathrm{R}$, et al. Six steps in quality intervention development (6SQuID). J Epidemiol Community Health 2015:jech2015-205952.

60. Hawkins J, Madden K, Fletcher A, et al. Development of a framework for the co-production and prototyping of public health interventions. BMC Public Health 2017;17:689.

61. European Commission/EACEA/Eurydice. Early childhood education and care systems in europe. national information sheets. Luxembourg: Publications Office of the European Union, 2015.

62. Horne PJ, Tapper K, Lowe CF, et al. Increasing children's fruit and vegetable consumption: a peer-modelling and rewards-based intervention. Eur J Clin Nutr 2004;58:1649-60.

63. Corsini N, Slater A, Harrison A, et al. Rewards can be used effectively with repeated exposure to increase liking of vegetables in 4-6-yearold children. Public Health Nutr 2013;16:942-51.

64. Wardle J, Cooke LJ, Gibson EL, et al. Increasing children's acceptance of vegetables; a randomized trial of parent-led exposure. Appetite 2003:40:155-62.

65. Davies G, Reilly JJ, McGowan AJ, et al. Validity, practical utility, and reliability of the activPAL ${ }^{\mathrm{TM}}$ in preschool children. Med Sci Sports Exerc 2012;44:761-8.

66. Martin A, McNeill M, Penpraze V, et al. Objective measurement of habitual sedentary behavior in pre-school children: comparison of activPAL With Actigraph monitors. Pediatr Exerc Sci 2011;23:468-76.

67. Harrington DM, Welk GJ, Donnelly AE. Validation of MET estimates and step measurement using the ActivPAL physical activity logger. J Sports Sci 2011;29:627-33.

68. Alghaeed Z, Reilly JJ, Chastin SF, et al. The influence of minimum sitting period of the ActivPAL ${ }^{\mathrm{TM}}$ on the measurement of breaks in sitting in young children. PLoS One 2013;8:e71854.

69. Cole TJ, Freeman JV, Preece MA. Body mass index reference curves for the UK, 1990. Arch Dis Child 1995;73:25-9.

70. de Beer M, Timmers T, Weijs PJM, et al. Validation of total body water analysis by bioelectrical impedance analysis with deuterium dilution in (pre)school children. E Spen Eur E J Clin Nutr Metab 2011;6:e223-6.

71. Mouratidou T, Miguel ML, Androutsos O, et al. Tools, harmonization and standardization procedures of the impact and outcome evaluation indices obtained during a kindergarten-based, familyinvolved intervention to prevent obesity in early childhood: the ToyBox-study. Obes Rev 2014;15(Suppl 3):53-60.

72. Kipping R, Jago R, Metcalfe C, et al. NAP SACC UK: protocol for a feasibility cluster randomised controlled trial in nurseries and at home to increase physical activity and healthy eating in children aged 2-4 years. BMJ Open 2016;6:e010622.

73. Castro FG, Barrera M, Martinez CR. The cultural adaptation of prevention interventions: resolving tensions between fidelity and fit. Prev Sci 2004;5:41-5.

74. ToyBox-Study. ToyBox - a European multi-country study to develop an obesity prevention programme specifically for pre-school children. Secondary ToyBox - a European multi-country study to develop an obesity prevention programme specifically for pre-school children. 2012. http://www.toybox-study.eu/?q=en/node/1 


\section{Correction: Adapting the ToyBox obesity prevention intervention for use in Scottish preschools: protocol for a feasibility cluster randomised controlled trial}

Malden S, Hughes AR, Gibson A, et al. Adapting the ToyBox obesity prevention intervention for use in Scottish preschools: protocol for a feasibility cluster randomised controlled trial. BMJ Open 2018;8:e023707. doi: 10.1136/bmjopen-2018-023707.

The original version of this manuscript contained an error in the 3rd affiliation:

Department of Movement and Sports Sciences, Ghent University, Ghent, UK

Should be:

Department of Movement and Sports Sciences, Ghent University, Ghent, Belgium

Open access This is an open access article distributed in accordance with the Creative Commons Attribution Non Commercial (CC BY-NC 4.0) license, which permits others to distribute, remix, adapt, build upon this work non-commercially, and license their derivative works on different terms, provided the original work is properly cited, appropriate credit is given, any changes made indicated, and the use is non-commercial. See: http://creativecommons.org/licenses/by-nc/4.0/.

(C) Author(s) (or their employer(s)) 2018. Re-use permitted under CC BY-NC. No commercial re-use. See rights and permissions. Published by BMJ.

BMJ Open 2018;9:e023707corr1. doi:10.1136/bmjopen-2018-023707corr1

A) Check for updates 